\title{
Comparison of CT and MRI in imaging diagnosis of aortic dissection
}

\author{
Jianing WANG ${ }^{1 *}$, Junmin $\mathrm{XIE}^{2 *}$, Xiaomin $\mathrm{MENG}^{2}$, Xuepeng GONG ${ }^{3 *}$
}

\begin{abstract}
Aortic dissection(AD) is a life-threatening disease due to a tear in the intimal layer of the aorta within the aortic wall. To compare diagnostic value and imaging of $\mathrm{AD}$ between computed tomography(CT) and magnetic resonance imaging (MRI). $120 \mathrm{AD}$ patients diagnosed were examined with 64-slice CT and 1.5 MRI, the imaging data of true and false lumen, intimal flap, intimal tear, mural thrombus and aortic calcification were compared. The intimal flap rate of CT and MRI was $81.7 \%$ and $100 \%$, respectively; The intimal tear rate of CT and MRI was $68.3 \%$ and $83.3 \%$, respectively; The rate of mural thrombus in CT and MRI was $26.7 \%$ and $54.2 \%$, respectively; The rate of aortic calcification in CT and MRI was $62.5 \%$ and $18.3 \%$, respectively; The number of patients with intimal tear lower than $1 \mathrm{~mm}$ in CT and MRI was 5 and 0 , respectively. Both CT and MRI can show the true and false lumen well, but the detection rate of intimal flap, intimal tear and mural thrombosis in MRI is significantly higher than that in CT, and the detection rate of aortic calcification and intimal tear $<1 \mathrm{~mm}$ in CT is higher than that in MRI.
\end{abstract}

Keywords: aortic dissection; CT; MRI; diagnosis value; imaging.

Practical Application: CT and MRI have their respective advantages in diagnosing aortic dissection.

\section{Introduction}

Cardio- cerebrovascular diseases have become a high-frequency disease. Common cardio- cerebrovascular diseases include cerebral hemorrhage, myocardial infarction, aortic aneurysms and aortic dissection (AD) (Omura et al., 2017; Acosta et al., 2019), in which the aortic dissection is also known as a dissection aneurysm, an aortic wall-media hematoma or a hemorrhage (Subramanya et al., 2018). The etiology of AD is not clear. The main risk factor for $\mathrm{AD}$ is high blood pressure, which causes high-pressure blood flow in the aortic lumen to be injected into the media to form a hematoma and extend the hematoma in the arterial wall (Liu et al., 2018). In recent years, the incidence of $\mathrm{AD}$ has increased from one in 100,000 per year to 10 in 100,000 per year (Guo et al., 2017a). Aortic dissection (AD) is very dangerous, if it is not diagnosed and treated on time. According to a recent report, without treatment, the hourly mortality rate of $\mathrm{AD}$ increases by $1 \%$ to $1.4 \%$, resulting in $68 \%$ mortality rate within $48 \mathrm{~h}$. Patients with type A dissection have the highest mortality rate, with a reported mortality rate of $58 \%$ in patients without surgery and $26 \%$ in patients with surgery. In contrast, patients with type B dissection have a lower mortality rate. The mortality rate of patients treated with medication is $11 \%$ lower than that of patients treated with surgery, and it is reported to be $31 \%$ (Pape et al., 2015). Therefore, in order to reduce the morbidity and mortality of patients with clinically suspected AD, early and accurate diagnosis and decision-making on surgery or conservative intervention is essential.
At present, imaging techniques computed tomography (CT) and magnetic resonance imaging (MRI) are the most commonly used methods for the diagnosis of AD (Hartnell, 2001; Liu \& Huang, 2018). For instance, hybrid PET-CT and 4D flow MRI may afford the non-invasive assessment of anatomic, hemodynamic, and molecular features of the aorta, and provide preventative surgical intervention for patients with $\mathrm{AD}$ (Adriaans et al., 2019). A wider understanding of the capabilities of CT and MRI and choosing the best protocols will further expand the use of these techniques and replace conventional angiography or digital subtraction angiography (DSA). Similar considerations apply when comparing the relative performance of imaging techniques reported in the scientific literature (Adriaans et al., 2019). Although one technique may be useful for establishing a diagnosis, it may not provide all of the information concerning the extent of aortic disease needed to make management decisions (Fuglsang et al., 2017). This article focuses on these CT and MRI methods, and compares the advantages of CT and MRI in the diagnosis of $\mathrm{AD}$.

\section{Materials and Methods}

\subsection{Patients}

From January 2017 to January 2019, a total of 120 patients including 65 males and 55 females, with an average age of $56.85 \pm$ 16.23 years with $\mathrm{AD}$ diagnosed in our hospital were selected for this study. Inclusion Criteria: (1) All patients were diagnosed as AD by DSA; (2) All patients had a history of hypertension. Exclusion 
criteria: (1) Patients with severe heart, liver and other important organ failure; (2) Patients with severe cognitive dysfunction, unable to cooperate with the examination; (3) Patients with hepatitis $\mathrm{B}$, syphilis, acquired immune deficiency syndrome (AIDS) and other infectious diseases. The 2-dimentional (2D) DSA was performed on a GE Innova 3100 system for diagnosis of patients with $\mathrm{AD}$. The images were taken in the anterior, posterior, and oblique $30^{\circ}$ projections (Lu et al., 2017). The obtained images were analyzed on the GE Advantage Workstation 4.3. The imaging criteria and rating method of DSA was as followed: (1) The AD type; (2) Morphology of true and false lumens, and the intimal flap detection; (3) The number of intimal entry tears, and the site and size of initial entry; (4) The involved condition of aorta branches.

\subsection{Methods}

All 120 patients were examined with 64-slice CT and 1.5MRI. Patients underwent plain and contrast enhanced CT examination. The CT scan was scanned with GE 64 row 128-slice CT. The thickness of the scanning slice was $5 \mathrm{~mm}$, the reconstruction slice was $0.5 \mathrm{~mm}$, the scanning voltage was $120 \mathrm{KV}$, and the scanning current was $320 \mathrm{~mA}$. The contrast agent was Iohexol. Contrast material was injected into the median cubital vein at the speed of $3.5 \mathrm{~mL} / \mathrm{s}$ by using a high pressure syringe, and scanning started 25 seconds later (Guo et al., 2017b; Li, 2017; Ren $\& \mathrm{Xu}, 2019)$. The MRI scan was performed using GE 1.5T MRI. The magnetic resonance spin echo scan was performed on the left front oblique position and the axial position. The layer thickness was $7.0 \mathrm{~mm}$, the scanning field of view (FOV) was 300 to $330 \mathrm{~mm}$, the echo time (TE) was $7.5 \mathrm{~ms}$, the repeat time (TR) was $114 \mathrm{~ms}$, left front oblique film parameter settings, slice distance was $2 \mathrm{~mm}$, slice thickness was $5.5 \mathrm{~mm}$, scanning field of view was 300 to $350 \mathrm{~mm}$, and TR/TE was $50 \mathrm{~ms} / 12 \mathrm{~ms}$. Gadolinium-DTPA (15 mL), a contrast agent, was injected intravenously into the median cubital vein (Bonfanti et al., 2018; Orabi et al., 2018; Renard et al., 2018; Yu, 2018). After the scan was finished, the blood flow in the large blood vessels of the heart was observed using a movie playback software. This retrospective study obtained the consent of the patients and the Ethics Committee of our hospital.

\subsection{Evaluation index}

The DeBakey classification was applied for the patients with $\mathrm{AD}$ in this study. The data including the true and false lumen, the detection rate of intimal flap, intimal tear, mural thrombosis, and aortic calcification were evaluated in accordance with the following imaging analysis criteria and rating methods (Bhamidipati \& Ailawadi, 2009; Tran \& Khoynezhad, 2009; Dehghan et al., 2017; Liu et al., 2017; Chun et al., 2018).

In the DeBakey classification, DeBakey type I AD is extensive and the break is in the ascending aorta; DeBakey type II AD is limited to the ascending portion of aorta; DeBakey type III $\mathrm{AD}$ is confined as a breach in the descending aorta, while type IIIa $\mathrm{AD}$ refers to the descending thoracic aorta, and type IIIb AD extends into the abdominal aorta. According to the International Registry of Acute Aortic Dissection (IRAD), the $\mathrm{AD}$ detection risk score of 0 to 3 is calculated on the basis of the number of risk categories (high-risk predisposing conditions, high-risk pain features, high-risk examination features) in which patients met criteria (Rogers et al., 2011). No high risk feature presented refers to score 0 , any single high risk category presented is score 1 , and two or three high risk categories presented is confined as score $2-3$.

\subsection{Statistical analysis}

The Statistical Product and Service Solutions (SPSS) statistics software version 20.0 (SPSS Inc., Chicago, IL, USA) was used to analyze the data. Results were compared using t or X2 examination. $\mathrm{P}<0.05$ was considered to be statistically significant.

\section{Results}

The patients with $\mathrm{AD}$ were examined by CT and MRI scanning. The representative $\mathrm{CT}$ imaging data of $\mathrm{AD}$ from a patient were shown in Figure 1. From the CT scanning, the linear low-density

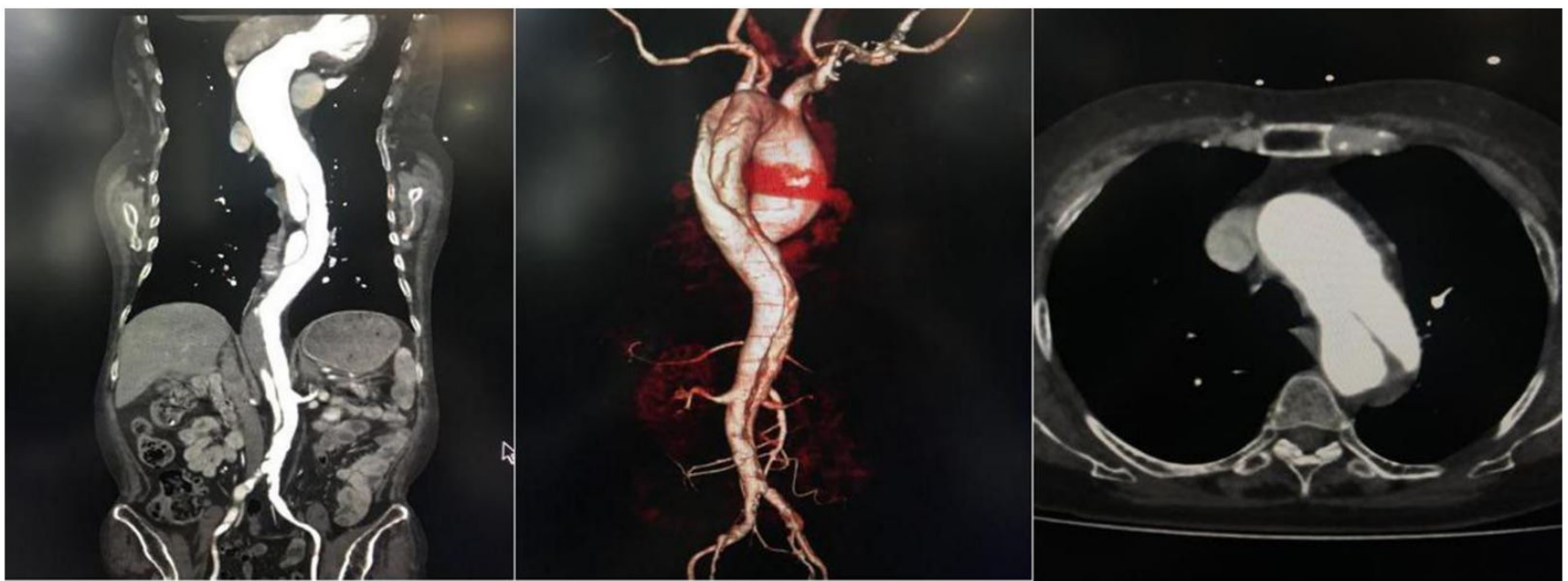

Figure 1. A representative CT imaging of patients with $\mathrm{AD}$. 
intima can be seen in the right external ilium artery, and aortic arch divides the lumen into real and false lumens. Meanwhile, Figure 2 showed the representative MR imaging of $\mathrm{AD}$. The contrast agent signals from the aortic arch to the upper end of the descending aortic line divide the descending aortic into two lumens in size. Collectively, DeBakey type I AD detected by CT and MRI were both performed in 25 patients with a detection rate of $20.83 \%(\mathrm{P}>0.05)$. DeBakey type II AD detected by CT and MRI were both performed in 30 patients with a detection rate of $25 \%(\mathrm{P}>0.05)$. DeBakey type III AD detected by CT and MRI were both 65 patients, and the detection rate was $54.17 \%$ ( $\mathrm{P}>0.05)$ (Table 1).

Both CT and MRI true and false cavity displays can reach $100 \%(\mathrm{P}>0.05)$; The intimal flap detection rate of CT and MRI is $81.7 \%$ and $100 \%$, respectively, and the results of the two groups are statistically significant $(\mathrm{X} 2=8.763, \mathrm{P}=0.001)$. Intimal tear film detection rate of CT and MRI reached $68.3 \%$ and $83.3 \%$ respectively, and the results of the two groups are statistically significant $(\mathrm{X} 2=8.759, \mathrm{P}=0.001)$. Compared to CT method, the mural thrombus film detection rate is enhanced in MRI method, and there are statistical significances between two

Table 1. Comparison of DeBakey types of aortic dissection between $\mathrm{CT}$ and MRI.

\begin{tabular}{lccc}
\hline Group & $\begin{array}{c}\text { DeBakey type I } \\
(\%)\end{array}$ & $\begin{array}{c}\text { DeBakey type II } \\
(\%)\end{array}$ & $\begin{array}{c}\text { DeBakey type III } \\
(\%)\end{array}$ \\
\hline CT & $25(20.83 \%)$ & $30(25 \%)$ & $65(54.17 \%)$ \\
MRI & $25(20.83 \%)$ & $30(25 \%)$ & $65(54.17 \%)$ \\
P & $>0.05$ & $>0.05$ & $>0.05$ \\
\hline
\end{tabular}

groups $(26.7 \%$ vs $54.2 \%, \mathrm{X} 2=6.621, \mathrm{P}=0.007)$. The $\mathrm{CT}$ aortic calcification film detection rate is $62.5 \%$, and the MRI aortic calcification detection rate is $18.3 \%$. The results of the two groups were statistically significant $(\mathrm{X} 2=12.683, \mathrm{P}=0.000)($ Table 2$)$.

There were 5 people with CT intimal tear $<1 \mathrm{~mm}$, and 0 with MRI intimal tear $<1 \mathrm{~mm}$. There is statistically significance between these two groups $(\mathrm{X} 2=4.782, \mathrm{P}=0.016)$ (Table 3$)$.

\section{Discussion}

Aortic dissection (AD) refers to the rupture of aortic intima or elastic fibropathy of media caused by various causes. The blood enters between the intima and media, which leads to the tear of media and the formation of double lumen aortic (Wen et al., 2017). AD can be attributed to the growing rise in obesity and food-linked diseases (Cottica et al., 2019; Maia et al., 2020). AD is common in middle-aged and elderly men, presents often with sudden severe chest pain, radiating to the back, abdomen, and sometimes even with shock (Rudnitskii \& Rudnytska, 2017). At present, there are many articles on the diagnosis of $\mathrm{AD}$ by CT and MRI (Nienaber et al., 1991; Melissano et al., 2012; Noorani et al., 2015; de Beaufort et al., 2019). The advantage of this paper lies in a more comprehensive and specific analysis of the classification and imaging manifestations of $\mathrm{AD}$, and further analyze the detection rate of rupture $<1 \mathrm{~mm}$, so as to fully evaluate the advantages and disadvantages of CT and MRI in the diagnosis of $\mathrm{AD}$.

Plain CT scan can show the calcification of avulsion intima to the aortic lumen. Contrast enhanced $\mathrm{CT}$ examination can show all kinds of signs of $\mathrm{AD}$, such as intimal flap, true and

Table 2. Comparison of CT and MRI aortic dissection.

\begin{tabular}{ccccccc}
\hline Group & $\mathrm{n}$ & True and False lumen & Intimal Flap & Entry of Tear & Mural Thrombus & Aortic Calcification \\
\hline CT & 120 & $120(100)$ & $98(81.7)$ & $82(68.3)$ & $32(26.7)$ & $75(62.5)$ \\
MRI & 120 & $120(100)$ & $120(100)$ & $100(83.3)$ & $65(54.2)$ & $22(18.3)$ \\
X2 & & 1.125 & 8.763 & 0.759 & 0.621 & 0.007 \\
P & & 0.237 & 0.001 & 0.01 & 0.000 \\
\hline
\end{tabular}
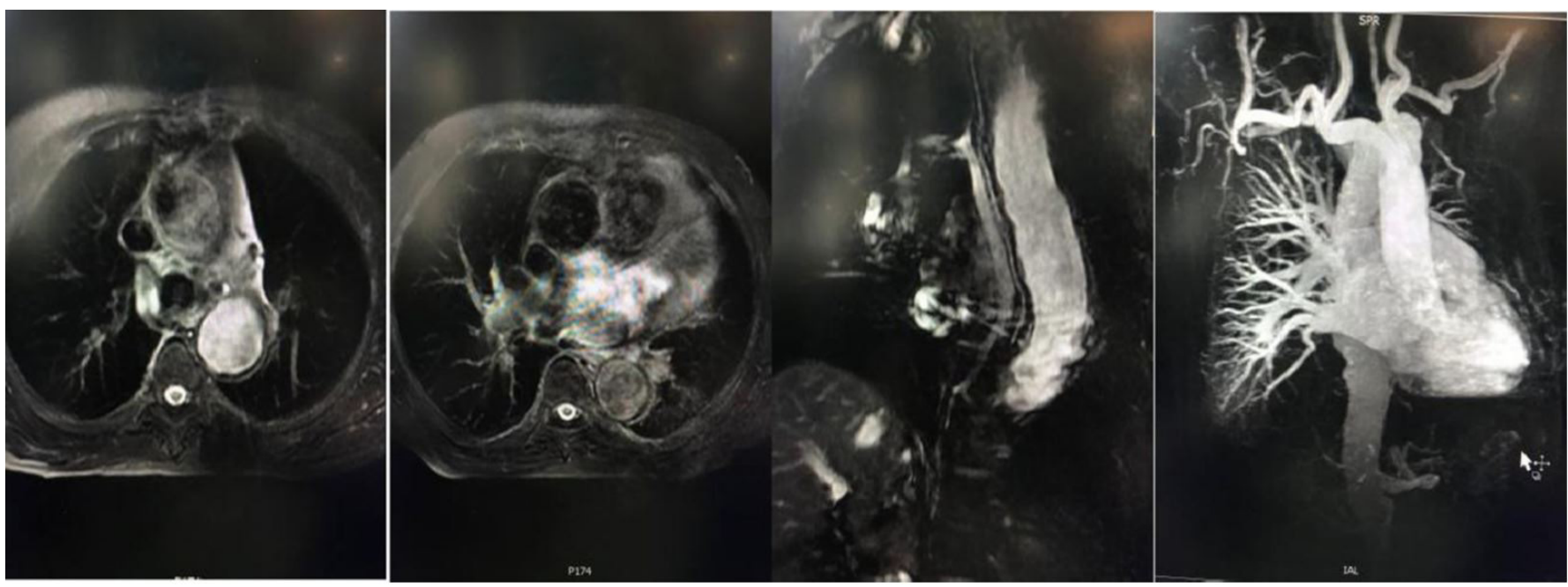

Figure 2. A representative MR imaging of patients with $\mathrm{AD}$. 
Table 3. Comparison of CT and MRI aortic dissection in intimal tear $<1 \mathrm{~mm}$.

\begin{tabular}{ccc}
\hline Group & $\mathrm{n}$ & Intimal tear $<1 \mathrm{~mm}$ \\
\hline CT & 120 & $5(4.17)$ \\
MRI & 120 & $0(0)$ \\
X2 & & 4.782 \\
P & & 0.016 \\
\hline
\end{tabular}

false cavity, mural thrombus, rupture and aortic calcification. When the contrast agent enters the false cavity, the line and strip transparent shadow can be seen between the true and false cavities, which is avulsion intima (Nienaber et al., 1991), and sometimes the filling defect is seen as mural thrombus (Noorani et al., 2015).

This study indicates that the detection rate of DeBakey typeI, type II, type III AD by CT and MRI is $20.83 \%, 25 \%, 54.17 \%$, respectively, which shows that there is no difference between CT and MRI in the diagnosis of AD. True and false lumen in CT and MRI can reach $100 \%$. Generally, the true lumen is narrow with fast blood flow, while the false lumen is wide with slow blood flow. A previous study suggested that CT enhanced scan can detect lumen dilatation in the affected segment and show the double-lumen aorta (Melissano et al., 2012). Four-dimensional (4D) MRI can reliably assess flow in the true and false lumens of the aorta in patients with $\mathrm{AD}$, because the true luminal blood flow is faster, presenting low or no signal, and the false luminal blood flow is slow, presenting low or medium signal (de Beaufort et al., 2019). On the MRI images, the true luminal is high signal, while the false luminal is low signal, so there is no significant difference between CT and MRI in the diagnosis of the true and false lumen.

In addition, this study found that, compared to CT, the detection rates of internal flap, intimal tear, mural thrombus in MRI were increased while the rates of aortic calcification are decreased significantly. Previous studies provided the evidence that internal flap, intimal tear, mural thrombus often occur in patients with $\mathrm{AD}$ and can be detected by $\mathrm{CT}$ and MRI (Yamada et al., 1995; Cowell et al., 2003; Huang et al., 2014; Kurabayashi et al., 2014; Zhao et al., 2020). However, when $\mathrm{AD}$ has a large scope, $\mathrm{CT}$ enhancement shows that the intima slices will spiral between the true and false lumens (Heuts et al., 2018), which may be the reason why the detection rate of the internal diaphragm in CT is lower than that in MRI. MRI can better show the jet sign of the intimal rupture (refers to the jet sign through the intimal rupture in the restricted signal flow space near the discontinuity of the internal diaphragm in the false cavity). MRI film can also show the jet sign, so the detection rate of intimal rupture in MRI is higher than that in CT. MRI examination of aortic dissection is based on the SE sequence. The axial plane is suitable for observing endometrial slices, true and false cavities, thrombus whereas left anterior oblique position shows the whole picture and interlayer. MRI can better show the intimal tear and contrast enhanced three-dimensional (3D) MRA images shows aortic involvement better. Therefore, MRI is better than CT in the endometrial film, intimal tear, and mural thrombus, and calcification is high density on CT, which is easier to identify. However, the low signal on MRI is difficult to identify, so the $\mathrm{CT}$ aortic calcification detection rate is higher than that of MRI. It can be seen from the data in Table 3 that there were 5 people with CT intimal tear $<1 \mathrm{~mm}$ and 0 with MRI intimal tear $<1 \mathrm{~mm}$. We analyze the reasons and believe that CT can reconstruct thin slices up to $0.5 \mathrm{~mm}$ and can form volume data. Therefore, the diagnosis rate of intimal tears $<1 \mathrm{~mm}$ is higher than that of MRI. However, this is a small cohort study on patients with aortic dissection. Further investigations with large size of patients as well as horizontal comparison of samples are still needed to verify the current findings.

\section{Conclusion}

In conclusion, this study proved that the detection rate of internal flap, intimal tear and mural thrombosis in MRI is significantly higher than that in CT, while the detection rate of aortic calcification and intimal rupture $<1 \mathrm{~mm}$ in $\mathrm{CT}$ is higher than that in MRI, indicating that CT and MRI have their respective advantages in diagnosing $\mathrm{AD}$.

\section{References}

Acosta, S., Kumlien, C., Forsberg, A., Nilsson, J., Ingemansson, R., \& Gottsäter, A. (2019). Engaging patients and caregivers in establishing research priorities for aortic dissection. SAGE Open Medicine, 7, 1-7. http://dx.doi.org/10.1177/2050312118822632. PMid:30637104.

Adriaans, B. P., Wildberger, J. E., Westenberg, J., Lamb, H. J., \& Schalla, S. (2019). Predictive imaging for thoracic aortic dissection and rupture: moving beyond diameters. European Radiology, 29(12), 6396-6404. http://dx.doi.org/10.1007/s00330-019-06320-7. PMid:31278573.

Bhamidipati, C. M., \& Ailawadi, G. (2009). Acute complicated and uncomplicated type III aortic dissection: an endovascular perspective. Seminars in Thoracic and Cardiovascular Surgery, 21(4), 373-386. http://dx.doi.org/10.1053/j.semtcvs.2009.11.008. PMid:20226352.

Bonfanti, M., Balabani, S., Alimohammadi, M., Agu, O., HomerVanniasinkam, S., \& Díaz-Zuccarini, V. (2018). A simplified method to account for wall motion in patient-specific blood flow simulations of aortic dissection: Comparison with fluid-structure interaction. Medical Engineering \& Physics, 58, 72-79.

Chun, J., Mansouri, M., Singh, A. (2018). Imaging of acute aortic conditions. In A. Singh (Ed.), Emergency radiology. Cham: Springer. http://dx.doi.org/10.1007/978-3-319-65397-6_1.

Cottica, S. M., Amado, D. A. V., Aguiar, S. C., Boeing, J. S., Franco, S. L., Zeoula, L. M., \& Visentainer, J. V. (2019). Antioxidant activity and lipid oxidation in milk from cows with soybean oil and propolis extract added to their feed. Food Science and Technology, 39(2), 467-474. http://dx.doi.org/10.1590/fst.33817.

Cowell, S. J., Newby, D. E., Burton, J., White, A., Northridge, D. B., Boon, N. A., \& Reid, J. (2003). Aortic valve calcification on computed tomography predicts the severity of aortic stenosis. Clinical Radiology, 58(9), 712-716. http://dx.doi.org/10.1016/S0009-9260(03)00184-3. PMid:12943644.

de Beaufort, H. W., Shah, D. J., Patel, A. P., Jackson, M. S., Spinelli, D., Yang, E. Y., Ghosn, M. G., Autry, K., Igo, S. R., Lumsden, A. B., Little, S. H., Trimarchi, S., \& Bismuth, J. (2019). Four-dimensional flow cardiovascular magnetic resonance in aortic dissection: Assessment in an ex vivo model and preliminary clinical experience. The Journal of Thoracic and Cardiovascular Surgery, 157(2), 467-476. http:// dx.doi.org/10.1016/j.jtcvs.2018.06.022. PMid:30121136. 
Dehghan, E., Wang, H., \& Syeda-Mahmood, T. (2017). Automatic detection of aortic dissection in contrast- enhanced ct. In IEEE 14th International Symposium on Biomedical Imaging (pp. 557-560). Melbourne, Australia: Institute of Electrical and Electronics Engineers.

Fuglsang, S., Heiberg, J., Hjortdal, V. E., \& Laustsen, S. (2017). Exercisebased cardiac rehabilitation in surgically treated type-A aortic dissection patients. Scandinavian Cardiovascular Journal, 51(2), 99-105. PMid:27808563.

Guo, J., Zhang, G., Tang, D., \& Zhang, J. (2017a). A case report of Takayasu arteritis with aortic dissection as initial presentation. Medicine, 96(45), e8610. http://dx.doi.org/10.1097/MD.0000000000008610. PMid:29137089.

Guo, W., Liu, Y., \& Yan, J. (2017b). Analysis of value of CTA in diagnosis of thoracic and abdominal aortic dissection. SYSTEMS MEDICINE, 2(11), 87-89.

Hartnell, G. G. (2001). Imaging of aortic aneurysms and dissection: CT and MRI. Journal of Thoracic Imaging, 16(1), 35-46. http://dx.doi. org/10.1097/00005382-200101000-00006. PMid:11149691.

Heuts, S., Adriaans, B. P., Gerretsen, S., Natour, E., Vos, R., Cheriex, E. C., Crijns, H., Wildberger, J. E., Maessen, J. G., Schalla, S., \& Sardari Nia, P. (2018). Aortic elongation part II: the risk of acute type A aortic dissection. Heart, 104(21), 1778-1782. http://dx.doi. org/10.1136/heartjnl-2017-312867. PMid:29593079.

Huang, C., Zhou, M., Liu, Z., Huang, D., Ran, F., Wang, W., Zhang, M., Liu, C., Liu, C., Qiao, T., \& Liu, C. (2014). Computed tomographybased study exploring the feasibility of endovascular treatment of type A aortic dissection in the Chinese population. Journal of Endovascular Therapy, 21(5), 707-713.

Kurabayashi, M., Okishige, K., Ueshima, D., Yoshimura, K., Shimura, T., Suzuki, H., Mitsutoshi, A., Aoyagi, H., Otani, Y., \& Isobe, M. (2014). Diagnostic utility of unenhanced computed tomography for acute aortic syndrome. Circulation Journal, 78(8), 1928-1934.

Li, H. (2017). Clinical value of 64 line 128 layer spiral CT and 1.5T MRI in aortic dissection. Clinical Cardiology, 33, 169-174.

Liu, D., Liu, J., Wen, Z., Li, Y., Sun, Z., Xu, Q., \& Fan, Z. (2017). 320row $C T$ renal perfusion imaging in patients with aortic dissection: a preliminary study. PLoS One, 12(2), e0171235. http://dx.doi. org/10.1371/journal.pone.0171235. PMid:28182709.

Liu, F., \& Huang, L. (2018). Usefulness of ultrasound in the management of aortic dissection. Reviews in Cardiovascular Medicine, 19(3), 103-109. PMid:31054559.

Liu, Y. J., Wang, X. Z., Wang, Y., He, R. X., Yang, L., Jing, Q. M., \& Liu, H. W. (2018). Correlation between sex and prognosis of acute aortic dissection in the Chinese population. Chinese Medical Journal, 131(12), 1430-1435. http://dx.doi.org/10.4103/0366-6999.233943. PMid:29893359.

Lu, D., Li, C. L., Lv, W. F., Ni, M., Deng, K. X., Zhou, C. Z., Xiao, J. K., Zhang, Z. F., \& Zhang, X. M. (2017). Diagnostic value of multislice computerized tomography angiography for aortic dissection: a comparison with DSA. Experimental and Therapeutic Medicine, 13(2), 405-412. http://dx.doi.org/10.3892/etm.2016.3985. PMid:28352308.

Maia, J. A. Jr., Henry, F. C., Della Lucia, S. M., Santos Junidr, A. C., Cabral, N. O., Quirini, C. R., \& Valle, F. R. A. F. (2020). Sensory attributes and Lipid oxidation of smoked lamb sausage formulated with passion fruit meal, potassium chloride and calcium chloride. Food Science and Technology, 40(2), 423-429. http://dx.doi.org/10.1590/fst.02419.

Melissano, G., Bertoglio, L., Rinaldi, E., Civilini, E., Tshomba, Y., Kahlberg, A., Agricola, E., \& Chiesa, R. (2012). Volume changes in aortic true and false lumen after the "PETTICOAT" procedure for type B aortic dissection. Journal of Vascular Surgery, 55(3), 641651. http://dx.doi.org/10.1016/j.jvs.2011.10.025. PMid:22285874.

Nienaber, C. A., Spielmann, R., Kodolitsch, Y., Siglow, V., Jaup, T., \& Bleifeld, W. (1991). Magnetic resonance imaging [MRI] or transesopha-geal echo [TEE] for anatomical mapping of thoracic aortic dissection? Journal of the American College of Cardiology, 17(2), A243. http://dx.doi.org/10.1016/0735-1097(91)91939-C.

Noorani, A., Kiessewetter, C., Botnar, R., Figueroa, C. A., \& Henningsson, M. (2015). Volumetric black-blood imaging of aortic dissection using T2 prepared inversion recovery. Journal of Cardiovascular Magnetic Resonance, 17(S1), P396. http://dx.doi.org/10.1186/1532429X-17-S1-P396.

Omura, A., Matsuda, H., Minami, H., Nakai, H., Henmi, S., Murakami, H., Yoshida, M., \& Mukohara, N. (2017). Early and late outcomes of operation for acute type a aortic dissection in patients aged 80 years and older. The Annals of Thoracic Surgery, 103(1), 131-138. http://dx.doi.org/10.1016/j.athoracsur.2016.05.046. PMid:27460914.

Orabi, N. A., Quint, L. E., Watcharotone, K., Nan, B., Williams, D. M., \& Kim, K. M. (2018). Distinguishing acute from chronic aortic dissections using CT imaging features. The International Journal of Cardiovascular Imaging, 34(11), 1831-1840. http://dx.doi.org/10.1007/ s10554-018-1398-x. PMid:29915877.

Pape, L. A., Awais, M., Woznicki, E. M., Suzuki, T., Trimarchi, S., Evangelista, A., Myrmel, T., Larsen, M., Harris, K. M., Greason, K., Di Eusanio, M., Bossone, E., Montgomery, D. G., Eagle, K. A., Nienaber, C. A., Isselbacher, E. M., \& O'Gara, P. (2015). Presentation, diagnosis, and outcomes of acute aortic dissection: 17-year trends from the international registry of acute aortic dissection. Journal of the American College of Cardiology, 66(4), 350-358. http://dx.doi. org/10.1016/j.jacc.2015.05.029. PMid:26205591.

Ren, H., \& Xu, Z. (2019). Clinical effect and evaluation of dual-source 64-slice CT and 1.5T MRI in aortic dissection. Mod Med Imagelogy, 28, 1017-1018.

Renard, Y., Hossu, G., Chen, B., Krebs, M., Labrousse, M., \& Perez, M. (2018). A guide for effective anatomical vascularization studies: useful ex vivo methods for both CT and MRI imaging before dissection. Journal of Anatomy, 232(1), 15-25. http://dx.doi.org/10.1111/ joa.12718. PMid:29023687.

Rogers, A. M., Hermann, L. K., Booher, A. M., Nienaber, C. A., Williams, D. M., Kazerooni, E. A., Froehlich, J. B., O'Gara, P. T., Montgomery, D. G., Cooper, J. V., Harris, K. M., Hutchison, S., Evangelista, A., Isselbacher, E. M., \& Eagle, K. A. (2011). Sensitivity of the aortic dissection detection risk score, a novel guideline-based tool for identification of acute aortic dissection at initial presentation: results from the international registry of acute aortic dissection. Circulation, 123(20), 2213-2218. http://dx.doi.org/10.1161/ CIRCULATIONAHA.110.988568. PMid:21555704.

Rudnitskii, A. G., \& Rudnytska, M. A. (2017). Segmentation and denoising of phase contrast MRI image of the aortic lumen via fractal and morphological processing. In IEEE 37th International Conference on Electronics and Nanotechnology (ELNANO) (pp. 344-348). Kiev, Ukraine: Institute of Electrical and Electronics Engineers.

Subramanya, V., Ambale-Venkatesh, B., Ohyama, Y., Zhao, D., Nwabuo, C. C., Post, W. S., Guallar, E., Ouyang, P., Shah, S. J., Allison, M. A., Ndumele, C. E., Vaidya, D., Bluemke, D. A., Lima, J. A., \& Michos, E. D. (2018). Relation of sex hormone levels with prevalent and 10year change in aortic distensibility assessed by MRI: the multi-ethnic study of atherosclerosis. American Journal of Hypertension, 31(7), 774-783. http://dx.doi.org/10.1093/ajh/hpy024. PMid:29471444. 
Tran, T. P., \& Khoynezhad, A. (2009). Current management of type B aortic dissection. Vascular Health and Risk Management, 5(1), 53-63. PMid:19436678.

Wen, D., Zhao, H., Duan, W., An, R., Li, J., \& Zheng, M. (2017). Combined $\mathrm{CT}$ angiography of the aorta and craniocervical artery: a new imaging protocol for assessment of acute type A aortic dissection. Journal of Thoracic Disease, 9(11), 4733-4742. http://dx.doi.org/10.21037/ jtd.2017.09.28. PMid:29268544.

Yamada, E., Matsumura, M., Kyo, S., \& Omoto, R. (1995). Usefulness of a prototype intravascular ultrasound imaging in evaluation of aortic dissection and comparison with angiographic study, transesophageal echocardiography, computed tomography, and magnetic resonance imaging. The American Journal of Cardiology, 75(2), 161-165. http:// dx.doi.org/10.1016/S0002-9149(00)80067-3. PMid:7810493.

Yu S. (2018). Comparison of clinical effects of 1.5T MRI and 64-row 128-slice spiral CT in aortic dissection. Mod Med Imagelogy, 27, 1958-1959.

Zhao, H., Ma, W., Wen, D., Duan, W., \& Zheng, M. (2020). Computed tomography angiography findings predict the risk factors for preoperative acute ischaemic stroke in patients with acute type $\mathrm{A}$ aortic dissection. European journal of cardio-thoracic surgery, 57(5), 912-919. 